\title{
A Scalar Relativistic Full-Potential LCAO Method
}

\author{
Shugo Suzuki and Kenji NAKaO \\ Institute of Materials Science, University of Tsukuba, Tsukuba 305-8573
}

(Received October 14, 1999 )

\begin{abstract}
We present a new scalar relativistic formulation for the full-potential linear-combinationof-atomic-orbitals method based on the density-functional theory. Three approximations are introduced to overcome computational difficulty. The first is to consider only the large component of the four-component spinor, neglecting the small component. The second is to neglect the energy dependence in the Hamiltonian reduced for the large component. The third is to replace the material-dependent potential with the atomic potential in relativistic corrections. After the three approximations, we identify the mass-velocity and Darwin terms and also the spin-orbit coupling, where the latter is to be omitted according to the definition of the scalar relativistic formulation. The computational effort of the present method is reduced considerably in comparison with that of the fully relativistic method, being almost the same as that of the nonrelativistic method. We apply the present method within the local-density approximation to several diatomic molecules with heavy elements, crystalline $\mathrm{Au}$, and crystalline InSb. The results are improved considerably in comparison with the nonrelativistic results. The calculated structural properties are in good agreement with the fully relativistic results and also with the experimental results. The calculated electronic properties are also improved considerably in comparison with the nonrelativistic results and are also in good agreement with the fully relativistic results except for the effect due to the spin-orbit coupling.
\end{abstract}

KEYWORDS: scalar relativistic calculations, full-potential calculations, LCAO method, density-functional theory, band calculations, structure optimizations 


\section{§1. Introduction}

The structural and electronic properties of materials with heavy elements are strongly affected by relativistic effects. Core electrons experience strong nuclear field and, consequently, the spatial extent of the core-electron orbitals is contracted substantially. As a result, the efficient screening of nuclear charge is caused by the core electrons, inducing the expansion of valence-electron orbitals, especially of $d$ and $f$ orbitals. ${ }^{1}$ ) This effect, which is known as the indirect relativistic effect, strongly affects the structural properties as well as the electronic properties. ${ }^{2)}$ The mass-velocity and Darwin terms are responsible for the indirect relativistic effect. Furthermore, of another importance is the spin-orbit coupling. For example, the splitting due to the spin-orbit coupling in the conduction and valence bands in crystals with heavy elements sometimes has the same order of magnitude as the crystal-field splitting.

The strategy which treats the mass-velocity and Darwin terms but neglects the spin-orbit coupling is known as the scalar relativistic method. The method has a practical advantage that the computational effort is reduced considerably in comparison with the fully relativistic method, which considers not only the mass-velocity and Darwin terms but also the spin-orbit coupling. So far, the scalar relativistic method has been implemented in several methods: the augmented-plane-wave $(\mathrm{APW})$ method, ${ }^{3)}$ the linearized muffin-tin-orbital (LMTO) method, ${ }^{4)}$ the Korringa-Kohn-Rostoker $(\mathrm{KKR})$ method, ${ }^{5)}$ and the linear-combination-of-atomic-orbitals (LCAO) method. ${ }^{6-18)}$ In particular, the usefulness of the scalar relativistic formulation given by Koelling and Harmon ${ }^{19)}$ for the APW, LMTO, and KKR method has been established in the last two decades. On the contrary, there are no standard scalar relativistic formulations for the LCAO method; it is difficult to apply directly the Koelling-Harmon formulation to the LCAO method because this method uses fixed basis sets in contrast to the APW, LMTO, and KKR methods.

One of several approaches for the scalar relativistic LCAO method is to use the FoldyWouthuysen-Tani (FWT) transformation, ${ }^{6-12)}$ where the mass-velocity and Darwin terms are treated perturbatively by an expansion in powers of the inverse of the square of the speed of light. Although this approach encountered a difficulty due to singular behavior of the resultant terms near nuclei, the frozen core approximation has circumvented this difficulty. ${ }^{11)}$ Another approach $^{13-17)}$ is to use Douglas-Kroll-Hess (DKH) transformation, ${ }^{20-22)}$ which generates no singular terms in contrast to the case of the FWT transformation. Also, there is an alternative approach which uses pseudopotential for simulating the scalar relativistic effects. ${ }^{18)}$

The purpose of the present study is to propose a new scalar relativistic formulation for the LCAO method, which is closely related to the Koelling-Harmon formulation. We develop our scalar relativistic full-potential LCAO (SFLCAO) method on the same basis as in our previous fully relativistic full-potential LCAO (FFLCAO ${ }^{23)}$ and nonrelativistic full-potential LCAO (NFLCAO) ${ }^{24)}$ methods. The present method does not use either FWT or DKH transformation and also does not 
use pseudopotential for simulating the scalar relativistic effects. We apply the SFLCAO method within the local-spin-density approximation (LSDA) to several diatomic molecules with heavy elements, crystalline $\mathrm{Au}$, and crystalline InSb. The calculated structural and electronic properties are compared with the FFLCAO and NFLCAO results as well as the experimental results. The SFLCAO method is described in $\S 2$ in detail. We next give the results of the application in $\S 3$. Finally, conclusions are given in $\S 4$.

\section{$\S 2 . \quad$ Method}

We begin with the Dirac-Kohn-Sham equations:

$$
\begin{gathered}
{\left[c \boldsymbol{\alpha} \cdot \boldsymbol{p}+(\beta-I) m c^{2}+V_{\mathrm{es}}(\boldsymbol{r})+V_{\mathrm{xc}}(\boldsymbol{r})+\beta \boldsymbol{\Sigma} \cdot \boldsymbol{B}_{\mathrm{xc}}(\boldsymbol{r})\right] \psi_{n}(\boldsymbol{r})=\varepsilon_{n} \psi_{n}(\boldsymbol{r}),} \\
\rho_{\mathrm{e}}(\boldsymbol{r})=\sum_{n} f_{n} \psi_{n}(\boldsymbol{r})^{*} \psi_{n}(\boldsymbol{r}),
\end{gathered}
$$

and

$$
\boldsymbol{m}_{\mathrm{e}}(\boldsymbol{r})=\sum_{n} f_{n} \psi_{n}(\boldsymbol{r})^{*} \beta \boldsymbol{\Sigma} \psi_{n}(\boldsymbol{r}) .
$$

In the Dirac operator in the left-hand side of eq. (1), $c$ and $m$ denote the speed of light and the rest mass of an electron, respectively, and the rest energy of an electron, $m c^{2}$, is subtracted. Also, $\boldsymbol{\alpha}$ and $\beta$ are the Dirac matrices in the usual representation. ${ }^{25)}$ In the Dirac-Kohn-Sham equations, the $n$th one-electron wave function, $\psi_{n}(\boldsymbol{r})$, is a four-component spinor. In eq. (1), $V_{\mathrm{es}}(\boldsymbol{r})$ is the electrostatic potential due to the nucleus charge density, $\rho_{\mathrm{n}}(\boldsymbol{r})$, and the electron charge density, $\rho_{\mathrm{e}}(\boldsymbol{r})$. Also, $V_{\mathrm{xc}}(\boldsymbol{r})$ is the spin-averaged exchange-correlation potential and $\boldsymbol{B}_{\mathrm{xc}}(\boldsymbol{r})$ is the exchangecorrelation magnetic field due to the spin magnetization density, $\boldsymbol{m}_{\mathrm{e}}(\boldsymbol{r})$, which is calculated by using $\boldsymbol{\Sigma}=I_{2} \otimes \boldsymbol{\sigma}$ where $I_{2}$ is the $2 \times 2$ unit matrix and $\boldsymbol{\sigma}$ are the usual $2 \times 2$ Pauli spin matrices. The electron charge density $\rho_{\mathrm{e}}(\boldsymbol{r})$ and the spin magnetization density $\boldsymbol{m}_{\mathrm{e}}(\boldsymbol{r})^{26-31)}$ are given with $\psi_{n}(\boldsymbol{r})$ and the occupation number of the $n$th level, $f_{n}$. The extension of the formulation given here to the corresponding method for solids is on the same line as in the FFLCAO and NFLCAO methods. ${ }^{23,24)}$

We now replace the four-component spinor $\psi_{n}(\boldsymbol{r})$ with a pair of two-component spinors, i.e., the large component, $\psi_{n}^{\mathrm{L}}(\boldsymbol{r})$, and the small component, $\psi_{n}^{\mathrm{S}}(\boldsymbol{r}):{ }^{32)}$

$$
\psi_{n}(\boldsymbol{r})=\left[\begin{array}{c}
\psi_{n}^{\mathrm{L}}(\boldsymbol{r}) \\
\psi_{n}^{\mathrm{S}}(\boldsymbol{r})
\end{array}\right] .
$$

Here we define a $2 \times 2$ matrix, $V(\boldsymbol{r})$, as follows:

$$
V(\boldsymbol{r})=V_{\mathrm{es}}(\boldsymbol{r})+V_{\mathrm{xc}}(\boldsymbol{r})+\boldsymbol{\sigma} \cdot \boldsymbol{B}_{\mathrm{xc}}(\boldsymbol{r})
$$


Then eq. (1) is decomposed into a pair of equations as follows:

$$
c \boldsymbol{\sigma} \cdot \boldsymbol{p} \psi_{n}^{\mathrm{S}}(\boldsymbol{r})=\left[\varepsilon_{n}-V(\boldsymbol{r})\right] \psi_{n}^{\mathrm{L}}(\boldsymbol{r})
$$

and

$$
c \boldsymbol{\sigma} \cdot \boldsymbol{p} \psi_{n}^{\mathrm{L}}(\boldsymbol{r})=2 M(\boldsymbol{r}) c^{2} \psi_{n}^{\mathrm{S}}(\boldsymbol{r})
$$

where we define

$$
M(\boldsymbol{r})=m+\frac{\varepsilon_{n}-\left[V(\boldsymbol{r})-2 \boldsymbol{\sigma} \cdot \boldsymbol{B}_{\mathrm{xc}}(\boldsymbol{r})\right]}{2 c^{2}} .
$$

By eliminating $\psi_{n}^{\mathrm{S}}(\boldsymbol{r})$, we obtain the following equation for $\psi_{n}^{\mathrm{L}}(\boldsymbol{r})$ :

$$
\left[\boldsymbol{\sigma} \cdot \boldsymbol{p} \frac{1}{2 M(\boldsymbol{r})} \boldsymbol{\sigma} \cdot \boldsymbol{p}+V(\boldsymbol{r})\right] \psi_{n}^{\mathrm{L}}(\boldsymbol{r})=\varepsilon_{n} \psi_{n}^{\mathrm{L}}(\boldsymbol{r}) .
$$

It should be noted that the relativistic corrections are entirely due to the second term in the right-hand side of eq. (8).

The first approximation introduced in the present formulation is to consider only the large component, neglecting the small component. That is, we attribute the entire norm to the large component. It is most likely that the small component does not play important roles in the structural and electronic properties of materials because it is negligible in outer region far from nuclei. Accordingly, since the focus of the study of materials is usually on the structural and electronic properties, this approximation seems appropriate for most applications.

Furthermore, the second approximation is to neglect the energy dependence in $M(\boldsymbol{r})$ to reduce the above eigenvalue problem (9) to the standard one. We replace $\varepsilon_{n}$ in $M(\boldsymbol{r})$ with a constant, $\varepsilon_{0}$. It is reasonable to choose $\varepsilon_{0}$ in the vicinity of the energy range of valence electrons for studying the structural and electronic properties of materials. We checked the dependence of the results of calculations on the choice of $\varepsilon_{0}$ by using $\varepsilon_{0}=0$ and $-10 \mathrm{eV}$ for several cases. Consequently, it was found that a typical change in the results is about $0.1 \%$. In the application given in the next section, we use $\varepsilon_{0}=0 \mathrm{eV}$ throughout.

We now introduce the third approximation as follows. In the LCAO method, we expand $\psi_{n}^{\mathrm{L}}(\boldsymbol{r})$ by atomic orbitals, $\chi_{p}(\boldsymbol{r})$. It is thus sufficient to know the results of the operation of

$$
\boldsymbol{\sigma} \cdot \boldsymbol{p} \frac{1}{2 M(\boldsymbol{r})} \boldsymbol{\sigma} \cdot \boldsymbol{p}
$$

onto $\chi_{p}(\boldsymbol{r})$. By observing that $\chi_{p}(\boldsymbol{r})$ is localized near the atom and oscillates substantially in the vicinity of the nucleus, we approximate the material dependent $2 \times 2$ matrix $V(\boldsymbol{r})-2 \boldsymbol{\sigma} \cdot \boldsymbol{B}_{\mathrm{xc}}(\boldsymbol{r})$ in $M(\boldsymbol{r})$ by the spherical spin-unpolarized potential, $V_{p}(r)$, of the atom to which $\chi_{p}(\boldsymbol{r})$ belongs:

$$
V(\boldsymbol{r})-2 \boldsymbol{\sigma} \cdot \boldsymbol{B}_{\mathrm{xc}}(\boldsymbol{r}) \simeq V_{p}(r)\left[\begin{array}{ll}
1 & 0 \\
0 & 1
\end{array}\right] .
$$


The above expression emphasizes that $V_{p}(r)$ is a multiple of the $2 \times 2$ unit matrix with respect to the spin index. Also, we understand that the origin of the coordinate is taken at the center of the atom. We also denote $M(\boldsymbol{r})$ approximated with the $V_{p}(r)$ by $M_{p}(r)$ as follows:

$$
M(\boldsymbol{r}) \simeq M_{p}(r)=m+\frac{\varepsilon_{0}-V_{p}(r)}{2 c^{2}} .
$$

Although this approximation ignores the material dependence and also the spin dependence of $V(\boldsymbol{r})-2 \boldsymbol{\sigma} \cdot \boldsymbol{B}_{\mathrm{xc}}(\boldsymbol{r})$, we show in the next section that it is an excellent approximation.

We finally arrive at the omission of the spin-orbit coupling, i.e., the scalar relativistic procedure by its definition. Since $V_{p}(r)$ and $M_{p}(r)$ commute with $\boldsymbol{\sigma}$, we can further decompose the operator (10) into two parts after some manipulations as follows:

$$
\boldsymbol{\sigma} \cdot \boldsymbol{p} \frac{1}{2 M_{p}(r)} \boldsymbol{\sigma} \cdot \boldsymbol{p}=-\boldsymbol{\nabla} \frac{1}{2 M_{p}(r)} \boldsymbol{\nabla}+\frac{1}{4 M_{p}(r)^{2} c^{2}} \boldsymbol{\sigma} \cdot\left[\boldsymbol{\nabla} V_{p}(r) \times \boldsymbol{p}\right] .
$$

The first operator represents the mass-velocity and Darwin terms and the second operator represents the spin-orbit coupling. We then omit the spin-orbit coupling according to the definition of the scalar relativistic formulation. By applying the first operator onto $\chi_{p}(\boldsymbol{r})$ expressed as $R_{p}(r) Y_{l m}(\theta, \phi)$, where $R_{p}(r)$ is the radial wave function and $Y_{l m}(\theta, \phi)$ is the spherical harmonics, we obtain the following result:

$$
\begin{aligned}
-\nabla \frac{1}{2 M_{p}(r)} \boldsymbol{\nabla}\left[R_{p}(r) Y_{l m}(\theta, \phi)\right]= & -\frac{1}{2 M_{p}(r)} \nabla^{2}\left[R_{p}(r) Y_{l m}(\theta, \phi)\right] \\
& -\nabla \frac{1}{2 M_{p}(r)} \cdot \nabla\left[R_{p}(r) Y_{l m}(\theta, \phi)\right] .
\end{aligned}
$$

The first term includes the mass-velocity term while the second term is the Darwin term. The atomic eigenvalue problem requires the above expression to be equal to

$$
\left[\varepsilon_{p}-V_{p}(r)\right] R_{p}(r) Y_{l m}(\theta, \phi) .
$$

Consequently, the scalar relativistic equation for $R_{p}(r)$ is given by

$$
\begin{array}{r}
-\frac{1}{2 M_{p}(r)}\left[\frac{\mathrm{d}^{2} R_{p}(r)}{\mathrm{d} r^{2}}+\frac{2}{r} \frac{\mathrm{d} R_{p}(r)}{\mathrm{d} r}-\frac{l(l+1)}{r^{2}} R_{p}(r)\right]-\frac{1}{4 M_{p}(r)^{2} c^{2}} \frac{\mathrm{d} V_{p}(r)}{\mathrm{d} r} \frac{\mathrm{d} R_{p}(r)}{\mathrm{d} r} \\
=\left[\varepsilon_{p}-V_{p}(r)\right] R_{p}(r) .
\end{array}
$$

This is just the Koelling-Harmon equation ${ }^{19)}$ except for the replacement of $\varepsilon_{p}$ in $M_{p}(r)$ with $\varepsilon_{0}$, indicating a close relation of the present formulation to the Koelling-Harmon formulation. We solve this equation to generate the atomic orbitals employed in the SFLCAO calculations.

We now complete the formulation by finding the expression for the matrix elements of the operator (10) according to the above three approximation as well as the omission of the spin-orbit coupling. We find

$$
\int \chi_{p}(\boldsymbol{r})^{*}\left[\boldsymbol{\sigma} \cdot \boldsymbol{p} \frac{1}{2 M(\boldsymbol{r})} \boldsymbol{\sigma} \cdot \boldsymbol{p} \chi_{q}(\boldsymbol{r})\right] \mathrm{d} \boldsymbol{r} \simeq-\int \chi_{p}(\boldsymbol{r})^{*}\left[\boldsymbol{\nabla} \frac{1}{2 M_{q}(r)} \boldsymbol{\nabla} \chi_{q}(\boldsymbol{r})\right] \mathrm{d} \boldsymbol{r}
$$


Since the operator (10) is Hermite, we also find

$$
\int\left[\boldsymbol{\sigma} \cdot \boldsymbol{p} \frac{1}{2 M(\boldsymbol{r})} \boldsymbol{\sigma} \cdot \boldsymbol{p} \chi_{p}(\boldsymbol{r})\right]^{*} \chi_{q}(\boldsymbol{r}) \mathrm{d} \boldsymbol{r} \simeq-\int\left[\boldsymbol{\nabla} \frac{1}{2 M_{p}(r)} \nabla \chi_{p}(\boldsymbol{r})\right]^{*} \chi_{q}(\boldsymbol{r}) \mathrm{d} \boldsymbol{r} .
$$

We thus obtain the approximation for the matrix elements of the operator (10) as follows:

$$
\frac{1}{2}\left\{-\int \chi_{p}(\boldsymbol{r})^{*}\left[\boldsymbol{\nabla} \frac{1}{2 M_{q}(r)} \boldsymbol{\nabla} \chi_{q}(\boldsymbol{r})\right] \mathrm{d} \boldsymbol{r}-\int\left[\boldsymbol{\nabla} \frac{1}{2 M_{p}(r)} \boldsymbol{\nabla} \chi_{p}(\boldsymbol{r})\right]^{*} \chi_{q}(\boldsymbol{r}) \mathrm{d} \boldsymbol{r}\right\} .
$$

By noting that the left-hand side of eq. (14) is equal to the form (15), we arrive at the final expression:

$$
\frac{1}{2}\left\{\int \chi_{p}(\boldsymbol{r})^{*}\left[\varepsilon_{q}-V_{q}(r)\right] \chi_{q}(\boldsymbol{r}) \mathrm{d} \boldsymbol{r}+\int\left[\varepsilon_{p}-V_{p}(r)\right] \chi_{p}(\boldsymbol{r})^{*} \chi_{q}(\boldsymbol{r}) \mathrm{d} \boldsymbol{r}\right\} .
$$

The above expression ensures the Hamiltonian matrix to be Hermite. It is also worthwhile to note that the expression becomes exact in the nonrelativistic limit, where $M(\boldsymbol{r})$ is simply a constant, $m$; the operator (10) is just the nonrelativistic kinetic-energy operator in this limit.

The actual calculations proceed as follows. We first generate the atomic orbitals used as the basis functions by solving eq. (16). We then calculate the structural and electronic properties of a material by performing entirely the same procedure as in the nonrelativistic calculations ${ }^{24)}$ except for the use of the atomic orbitals generated as described above. Thus, the computational effort of the SFLCAO method is almost the same as that of the NFLCAO method except for a slight increase in the effort for generating the atomic orbitals, which is, however, a quite easy task in comparison with the self-consistent calculations of the material. The computational effort is thus reduced significantly in comparison with that of the FFLCAO method; the reduction is about one sixteenth. Furthermore, it is worthwhile to explain how to calculate the dissociation energy of a molecule or the cohesive energy of a crystal. By applying the same level of approximation for the relativistic effects to the material and to the constituent atoms, we calculate the dissociation or cohesive energy by subtracting the sum of the total energies of the constituent atoms from the total energy of the material. This procedure reduces the error in the dissociation or cohesive energy considerably. That is, the large error in the total energy associated with the core electrons in the SFLCAO and NFLCAO method is canceled out by the above subtraction despite that the difference among the FFLCAO, SFLCAO, and NFLCAO total energies exceeds $10000 \mathrm{eV}$ per atom for some cases.

\section{§3. Application}

We first apply the SFLCAO method to diatomic molecules with heavy elements; HfO, TaO, $\mathrm{IrC}, \mathrm{PtO}, \mathrm{Au}_{2}, \mathrm{HgH}, \mathrm{TlH}, \mathrm{TlF}, \mathrm{TlCl}, \mathrm{TlBr}, \mathrm{PbS}$, and $\mathrm{BiO}$. We do not study diatomic molecules containing W, Re, and Os because we cannot find the experimental data for the three elements. The results are compared with experimental results as well as the FFLCAO and NFLCAO results. 
The atomic orbitals employed are shown in Table I. The number of mesh points for each atom is also shown. We choose the atomic orbitals so that they have enough variational flexibility. That is, we use not only the atomic orbitals of neutral atoms but also those of charged atoms. We use a single orbital for each core orbital while double orbitals for each valence orbital. Double valence orbitals are necessary for describing the contraction of atomic orbitals accompanied with cohesion. Furthermore, a set of polarization functions is used for each atom, e.g., $2 p$ orbitals for $\mathrm{H}$ and $3 d$ orbitals for $\mathrm{C}, \mathrm{O}$, and $\mathrm{F}$, etc. Furthermore, the exchange-correlation functional within LSDA employed here is the Perdew-Zunger parameterization of Ceperley and Alder results. ${ }^{33,34)}$ Also, we perform the multipolar expansion of the electrostatic potential up to $3 .^{24)}$

The calculated bond lengths are shown in Table II with the experimental results. The FFLCAO and NFLCAO results are also shown. The agreement of the SFLCAO results with the experimental results is good; the maximum error of the SFLCAO results relative to the experimental results is $+2.5 \%$, which is for TlH. Also, the agreement of the SFLCAO results with the FFLCAO results is good; the maximum error of the SFLCAO results relative to the FFLCAO results is $+1.5 \%$, which is for $\mathrm{HgH}$. Furthermore, the comparison with the NFLCAO results shows substantial improvement on the calculated bond lengths; the errors of about $10 \%$ for $\mathrm{PtO}$ and $\mathrm{Au}_{2}$ are reduced to about 1 $\%$. These results indicate that molecular structures are mainly affected by the mass-velocity and Darwin terms but not by the spin-orbit coupling.

Table III shows the calculated spin polarizations as well as the experimental results deduced from the experimental spin multiplicity of the ground states of the molecules. ${ }^{35)}$ The FFLCAO and NFLCAO results are also shown. The SFLCAO calculations as well as the FFLCAO calculations succeed in reproducing the experimental spin polarizations. In particular, $\mathrm{TaO}, \mathrm{IrC}, \mathrm{HgH}$, and $\mathrm{BiO}$ have non-zero spin polarization in the ground states. It is interesting to note that the spin polarizations obtained by the SFLCAO method is exactly one half while the FFLCAO results are slightly smaller than one half. The reason for this is that spin is a good quantum number in the SFLCAO method while it is not in the FFLCAO method because of the spin-orbit coupling. In contrast to the success of the SFLCAO and FFLCAO calculations, the NFLCAO calculations give wrong results for $\mathrm{HfO}, \mathrm{TaO}$, and $\mathrm{PtO}$. The results thus implies that entire neglect of the relativistic effects is dangerous for materials with heavy elements because such neglect can result in wrong ground states for some cases.

We show the calculated one-electron energies of the highest-occupied and lowest-unoccupied molecular orbitals as well as the gaps between them in Table IV. The SFLCAO results agree with the FFLCAO results within about $0.5 \mathrm{eV}$ although the deviation of about $0.5 \mathrm{eV}$ is too large to be neglected. This means that the effect of the spin-orbit coupling on one-electron energies is considerable in contrast to its minor role in structural properties. The NFLCAO results are poor; the difference exceeds about $1 \mathrm{eV}$ for some molecules. This is not surprising because the NFLCAO 
method sometimes fails to give a correct ground state as pointed out above.

We finally show the calculated dissociation energies as well as the experimental results in Table V. It is found that chemical trend is reproduced to some extent. We cannot, however, find the reason for the non-systematic behavior that the FFLCAO results are largest for some molecules while the SFLCAO or NFLCAO results are largest for other molecules. Furthermore, there are noticeable overestimations relative to the experimental results in almost all of the calculated results; the exception is the underestimation for $\mathrm{Au}_{2}$ where the relativistic effects are most pronounced. We believe that the overestimation is due to LSDA. It is, however, necessary to study the nonsystematic behavior in the dissociation energy as well as the overestimation due to LSDA in the future.

We next study crystalline Au and InSb by using the SFLCAO method as well as the FFLCAO and NFLCAO methods. The conditions for the calculations are entirely the same as in our previous paper for comparison. ${ }^{23}$ ) The exchange-correlation energy functional within LSDA given by von Barth and Hedin is employed. We use 3096 and 2064 mesh points per atom for three-dimensional numerical integration in real space for $\mathrm{Au}$ and $\mathrm{InSb}$, respectively. Also, we use $185 \mathrm{k}$ points generated with the good-lattice-point method $^{36)}$ in the full Brillouin zone for Au while $16 \mathbf{k}$ points generated with the special-point method $^{37)}$ also in the full Brillouin zone for InSb. Furthermore, we perform the multipolar expansion of the electrostatic potential up to $8 .^{24)}$ The atomic orbitals used for $\mathrm{Au}$ are the same as in Table I. Also, the atomic orbitals used for InSb are $1 s, 2 s, 2 p, 3 s$, $3 p, 3 d, 4 s, 4 p, 4 d, 5 s$, and $5 p$ atomic orbitals of neutral In and $\mathrm{Sb}$ atoms and $5 s, 5 p$, and $5 d$ atomic orbitals of $\mathrm{In}^{2+}$ and $\mathrm{Sb}^{2+}$ atoms.

The results of the structure optimization for Au are shown in Table VI. The lattice constant and the bulk modulus obtained by the SFLCAO method are given as well as the FFLCAO and NFLCAO results. The results of experimental and other theoretical studies are also shown. The agreement of the lattice constant obtained by the SFLCAO method with the experimental one is good. The result is also in good agreement with the FFLCAO result. The bulk modulus calculated by the SFLCAO method is, on the other hand, slightly larger than the FFLCAO result. Since the present calculations are entirely the same except for the level of approximation for the relativistic effects, we believe that the small difference between the two results is still meaningful. The reason for this difference is, however, not clear. The agreement between the theoretical results is also good not depending whether the spin-orbit coupling is included or not. This again indicates that the spin-orbit coupling does not affect structural properties so much. On the other hand, the lattice constant calculated by the NFLCAO method is substantially overestimated and the bulk modulus is substantially underestimated. Its origin is apparently the entire neglect of the relativistic effects. The deficiency of the nonrelativistic calculations is also supported by another nonrelativistic result shown in Table VI. ${ }^{17)}$ 
The results of the band calculations by the FFLCAO, SFLCAO, and NFLCAO methods are shown in Figs. 1, 2, and 3, respectively. There is noticeable splitting due to the spin-orbit coupling in the FFLCAO result. On the contrary, there is no corresponding splitting in the SFLCAO result because the SFLCAO method neglects the spin-orbit coupling by its definition. It should, however, be noted that the mass-velocity and Darwin terms affect the electronic structure strongly. That is, by comparing the SFLCAO and NFLCAO results, it is found that the mass-velocity and Darwin terms make the $s$ band deep while the $d$ band shallow. This is the main reason for the contraction of the lattice constant calculated by the SFLCAO method in comparison with the NFLCAO result. The atomic origin of this effect is that $s$ orbitals experience the relativistic effects more strongly than $d$ orbitals and, thus, $s$ orbitals are contracted in comparison with $d$ orbitals. The contraction of $s$ orbitals results in the contraction of the lattice constant. Furthermore, the contraction of $s$ orbitals induces strong screening of nuclear charge and, consequently, results in the expansion of $d$ orbitals. This is the indirect relativistic effect and the origin of the relativistic effects on the structural properties shown above.

We also show the $d$-band width in Table VII, which is defined as the difference between the first and the fifth energy band at the $X$ point as shown in Figs. 1, 2, and 3. The SFLCAO result, 5.55 $\mathrm{eV}$, differs substantially from the FFLCAO result, $6.28 \mathrm{eV}$. This is also due to the neglect of the spin-orbit coupling, supported also by comparing with other theoretical results with the spin-orbit coupling. ${ }^{39,45)}$ It is also found that the effects due to the mass-velocity and Darwin terms cannot be neglected; the SFLCAO result is substantially larger than the NFLCAO result, $5.02 \mathrm{eV}$, as well as than another nonrelativistic result, $5.01 \mathrm{eV} .{ }^{17)}$ It is, however, found that there is a difference of about $0.3 \mathrm{eV}$ between the SFLCAO result and the other scalar relativistic results, $5.89 \mathrm{eV}^{43)}$ and $5.85 \mathrm{eV} .{ }^{17)}$ Since the present scalar relativistic formulation differs from the others to some extent, there can be an error of about $0.3 \mathrm{eV}$. The origin of the discrepancy is, however, not clear.

In Table VIII, we show the calculated cohesive energies as well as the experimental cohesive energy. The FFLCAO and SFLCAO methods overestimate the cohesive energy while the NFLCAO method underestimates it; a similar behavior is found in the calculated dissociation energies of $\mathrm{Au}_{2}$ shown in Table V. It is most likely that the origin of the overestimation in the FFLCAO and SFLCAO results is due to LSDA while the origin of the underestimation in the NFLCAO result is due to the entire neglect of the relativistic effects. The agreement between the FFLCAO and SFLCAO results is moderate; there is a difference of about $0.2 \mathrm{eV}$ probably due to the effect of the spin-orbit coupling. On the other hand, there is a noticeable difference of about $1 \mathrm{eV}$ between the FFLCAO or SFLCAO result and the NFLCAO result. This also implies the importance of the relativistic effects in $\mathrm{Au}$.

We next show the results of the structure optimization of InSb by the SFLCAO method as well as the FFLCAO and NFLCAO results in Table IX. The results of experimental and other theo- 
retical studies are also shown. The lattice constant calculated by the SFLCAO method is in good agreement with both the experimental and FFLCAO results. On the other hand, there is a slight overestimation in the NFLCAO result. Since the present calculations are entirely the same except for the level of approximation for the relativistic effects, we believe that the small overestimation by the NFLCAO method is due to the neglect of the relativistic effects. Also, the agreement of the SFLCAO result with the result of the fully relativistic full-potential KKR calculations ${ }^{39)}$ and also with the result of the scalar relativistic full-potential linearized APW calculations ${ }^{3)}$ is good. Although there is a slight underestimation in the results of the pseudopotential plane-wave calculations, ${ }^{3,46)}$ this underestimation is attributed to the neglect of the partial core correction. ${ }^{3)}$ We also show experimental and theoretical bulk modulus in Table IX. The agreement between the results is good although a slight overestimation is found in the NFLCAO result. According to the same reason mentioned above, we also believe that the slight overestimation is due to the neglect of the relativistic effects. The results for the lattice constant and the bulk modulus indicate that the relativistic effects do not affect these quantities of InSb so much in comparison with the case of $\mathrm{Au}$. This is reasonable because $\mathrm{In}$ and $\mathrm{Sb}$ are much lighter than $\mathrm{Au}$.

The results of the band calculations for InSb by the FFLCAO, SFLCAO, and NFLCAO methods are shown in Figs. 4, 5, and 6, respectively. The overall agreement between the FFLCAO and SFLCAO results is good although there are noticeable splitting due to the spin-orbit coupling in the FFLCAO result. On the other hand, the difference between the FFLCAO or SFLCAO result and the NFLCAO result is remarkable. The most noticeable point is that there exists a gap of 1.0 $\mathrm{eV}$ in the NFLCAO result while there exist no gaps in the FFLCAO and SFLCAO results. The gap obtained by the NFLCAO method is larger than the experimental one, $0.2 \mathrm{eV}$. This implies that the NFLCAO gap is artificial because LSDA always underestimates gaps in semiconductors and insulators. In fact, it is known that LSDA fails to give a gap for InSb; in agreement with the present result, there are no gaps in the results of the fully relativistic full-potential KKR calculations, ${ }^{39)}$ where LSDA with the same exchange-correlation energy functional as in the present calculations is employed. The result again emphasizes the importance of the relativistic effects to electronic properties in contrast to their minor roles in structural properties.

We also show the one-electron energies at the $\Gamma, X$, and $L$ points at the experimental lattice constant obtained by the present study and those obtained by other theoretical studies in Table $\mathrm{X}$. The SFLCAO result is in good agreement with another scalar relativistic result shown in the table $^{3)}$ in spite of the apparent difference in the formulation; the maximum difference is about 0.2 $\mathrm{eV}$. The agreement with the FFLCAO result as well as the other theoretical results which consider the spin-orbit coupling is also good except for the effect due to the spin-orbit coupling. On the contrary, the NFLCAO result is poor, especially about the existence of the artificial gap in the NFLCAO result as already pointed out. 
We finally show the calculated cohesive energies as well as the experimental cohesive energy in Table XI. The situation is quite different from the case of Au. The FFLCAO method does not overestimate the cohesive energy but gives almost the same magnitude as the experimental result. On the contrary, the NFLCAO result overestimates it. The only agreement with the case of $\mathrm{Au}$ is the overestimation in the SFLCAO result. The fact that NFLCAO results are the largest is also found in the dissociation energies for some molecules as shown in Table $\mathrm{V}$ although the reason is not clear. Furthermore, the reason why the FFLCAO method does not overestimate the cohesive energy in spite of the use of LSDA is also not clear. Thus, it seems better not to conclude that the overestimation in the SFLCAO and NFLCAO results is due to LSDA. We cannot find a reason for these behavior and leave a detailed analysis to further studies.

\section{$\S 4$. Conclusions}

We have proposed a new scalar relativistic formulation for the LCAO method. The three approximation have been introduced to overcome computational difficulty. The first is to consider only the large component in the four-component spinor, neglecting the small component. The second is to neglect the energy dependence in the Hamiltonian reduced for the large component. The third is to replace the material-dependent potential with the atomic potential in the relativistic corrections. We then identify the mass-velocity and Darwin terms and also the spin-orbit coupling, where the latter is omitted according to the definition of the scalar relativistic formulation. The expression for the matrix elements for the scalar relativistic effects is also given.

The results of application within LSDA of the present scalar relativistic full-potential method, the SFLCAO method, are given, compared with the experimental results. Furthermore, we compare the SFLCAO results with the results obtained by the fully relativistic full-potential LCAO method, the FFLCAO method, and also with the results obtained by the nonrelativistic full-potential LCAO method, the NFLCAO method.

We first study several diatomic molecules with heavy elements: $\mathrm{HfO}, \mathrm{TaO}, \mathrm{IrC}, \mathrm{PtO}, \mathrm{Au}_{2}$, $\mathrm{HgH}, \mathrm{TlH}, \mathrm{TlF}, \mathrm{TlCl}, \mathrm{TlBr}, \mathrm{PbS}$, and $\mathrm{BiO}$. The calculated bond lengths are in good agreement with the experimental results as well as the FFLCAO results. It has been pointed out that the agreement between the SFLCAO and FFLCAO results means that the spin-orbit coupling plays a minor role in structural properties. On the other hand, the results differ from the NFLCAO results to considerable amount for some molecules. This indicates the importance of the relativistic effects, especially the scalar-relativistic effects, in studying structural properties. The spin polarizations of the molecules are also given. The results show that the SFLCAO method as well as the FFLCAO method reproduces experimental spin polarizations while the NFLCAO method gives wrong results for some molecules, also indicating the importance of the relativistic effects. The calculated one-electron energies of the highest-occupied and lowest-unoccupied molecular orbitals are compared next. The overall agreement between the SFLCAO and FFLCAO results is good although 
the difference of about $0.5 \mathrm{eV}$ is found probably due to the spin-orbit coupling. On the other hand, there exist noticeable deviations from the NFLCAO results. The dissociation energies of the molecules are also given. We have find noticeable overestimation probably due to LSDA although we could not find a reason for non-systematic behavior according to the level of approximation for the relativistic effects.

The results of application of the SFLCAO method to crystalline $\mathrm{Au}$ and InSb are given next. The calculated lattice constants and the bulk moduli are in good agreement with the experimental results as well as the FFLCAO results. The agreement with the NFLCAO results is, however, not good, especially for Au. The results also imply the importance of the relativistic effects, especially of the scalar relativistic effects, to structural properties of materials with heavy elements. The results of the band calculations are also given. The agreement between the SFLCAO and FFLCAO results is good except for the effect due to the spin-orbit coupling. We also give the calculated cohesive energies. In contrast to the good agreement in the lattice constants and the bulk moduli with the experimental results, there exist noticeable deviations in the calculated results probably due to LSDA. The detailed study of the non-systematic behavior according to the level of approximation for the relativistic effects is, however, open to further studies.

\section{Acknowledgements}

This work is supported in part by the Grant-in-Aid for Scientific Research on the Priority Area "Fullerenes and Nanotubes" by the Ministry of Education, Science, Sports and Culture of Japan. 


\section{References}

1) A. J. Freeman and J. P. Desclaux: J. Magn. Magn. Mater. 12 (1979) 11.

2) T. Ziegler, J. G. Snijders, and E. J. Baerends: J. Chem. Phys. 74 (1981) 1271.

3) For example, see S. Massidda, A. Continenza, A. J. Freeman, T. M. de Pascale, and F. Meloni: Phys. Rev. B 41 (1990) 12079.

4) For example, see T. Korhonen, M. J. Puska, and R. M. Nieminen: Phys. Rev. B 51 (1995) 9526.

5) For example, see M. Battocletti, H. Ebert, and H. Akai: Phys. Rev. B 53 (1996) 9776.

6) T. Ziegler, V. Tschinke, E. J. Baerends, J. G. Snijders, and W. Ravenek: J. Phys. Chem. 93 (1989) 3050.

7) J. Li, G. Schreckenbach, and T. Ziegler: J. Phys. Chem. 98 (1994) 4838.

8) J. Li, G. Schreckenbach, and T. Ziegler: J. Am. Chem. Soc. 117 (1995) 486.

9) J. Li, G. Schreckenbach, and T. Ziegler: Inorg. Chem. 34 (1995) 3245.

10) G. Schreckenbach, J. Li, and T. Ziegler: Int. J. Quantum Chem. Symp. 56 (1995) 477.

11) G. Schreckenbach and T. Ziegler: Int. J. Quantum Chem. 61 (1997) 899.

12) H. Jacobsen, G. Schreckenbach, and T. Ziegler: J. Phys. Chem. 98 (1995) 11406.

13) N. Rösch and O. D. Häberlen: J. Chem. Phys. 96 (1992) 6322.

14) O. D. Häberlen and N. Rösch: Chem. Phys. Lett. 199 (1992) 491.

15) N. J. M. Geipel and B. A. Hess: Chem. Phys. Lett. 273 (1997) 62.

16) J. C. Boettger: Int. J. Quantum Chem. 65 (1997) 565.

17) J. C. Boettger: Phys. Rev. B 57 (1998) 8743.

18) B. Delley: Int. J. Quantum Chem. 69 (1998) 423.

19) D. D. Koelling and B. N. Harmon: J. Phys. C: Solid State Phys. 10 (1977) 3107.

20) M. Douglas and N. M. Kroll: Ann. Phys. (N.Y.) 82 (1974) 89.

21) B. A. Hess: Phys. Rev. A 33 (1986) 3742.

22) G. Jansen and B. A. Hess: Phys. Rev. A 39 (1989) 6016.

23) S. Suzuki and K. Nakao: J. Phys. Soc. Jpn. 68 (1999) 1982.

24) S. Suzuki and K. Nakao: J. Phys. Soc. Jpn. 66 (1997) 3881.

25) W. Greiner: Relativistic Quantum Mechanics (Springer-Verlag, Berlin, 1990).

26) W. Gordon: Z. Phys. 50 (1928) 630.

27) A. H. MacDonald and S. H. Vosko: J. Phys. C: Solid State Phys. 12 (1979) 2977.

28) H. Ebert: Phys. Rev. B 38 (1988) 9390.

29) H. J. F. Jansen: Phys. Rev. B 38 (1988) 8022.

30) J. Forstreuter, L. Steinbeck, M. Richter, and H. Eschrig: Phys. Rev. B 55 (1997) 9415.

31) H. Yamagami, A. Mavromaras, and J. Kübler: J. Phys. Condens. Matter 9 (1997) 10881.

32) L. I. Schiff: Quantum Mechanics (McGraw-Hill, Tokyo, 1968).

33) J. P. Perdew and A. Zunger: Phys. Rev. B 23 (1981) 5048.

34) D. M. Ceperley and B. J. Alder: Phys. Rev. lett. 45 566(1980) 566.

35) K. P. Huber and G. Herzberg: Constants of Diatomic Molecules, Vol. 4, Molecular Spectra and Molecular Structure (Van Nostrand, New York, 1979).

36) L.-K. Hua and Y. Wang: Applications of Number Theory to Numerical Analysis (Springer-Verlag, Berlin, 1981).

37) D. J. Chadi and M. L. Cohen: Phys. Rev. B 8 (1973) 5747.

38) Numerical Data and Functional Relationships in Science and Technology - Crystal and Solid State Physics, Vol. 17a of Landolt-Börnstein, edited by O. Madelung (Springer, Berlin, 1984). 
39) S. Bei der Kellen and A. J. Freeman: Phys. Rev. B 54 (1996) 11187.

40) M. Methfessel and J. Kübler: J. Phys. F 12, (1982) 141.

41) A. Khein, D. J. Singh, and C. J. Umrigar: Phys. Rev. B 51 (1995) 4105.

42) M. J. Mehl and D. A. Papaconstantopoulos: Phys. Rev. B 54 (1996) 4519.

43) M. Sigalas, D. A. Papaconstantopoulos, and N. C. Bacalis: Phys. Rev. B 45 (1992) 5777.

44) C. Elsässer, N. Takeuchi, K. M. Ho, C. T. Chan, P. Braun, and M. Fahnle: J. Phys. Condens. Matter 2 (1990) 4371.

45) A. H. MacDonald, J. M. Daams, S. H. Vosko, and D. D. Koelling: Phys. Rev. B 25 (1982) 713.

46) S. B. Zhang and M. L. Cohen: Phys. Rev. B 35 (1987) 7604. 


\section{Figure Captions}

Fig. 1. Band structure of Au calculated by the fully relativistic full-potential LCAO method at the experimental lattice constant. The dotted line represents the Fermi level.

Fig. 2. Band structure of Au calculated by the scalar relativistic full-potential LCAO method at the experimental lattice constant. The dotted line represents the Fermi level.

Fig. 3. Band structure of Au calculated by the nonrelativistic full-potential LCAO method at the experimental lattice constant. The dotted line represents the Fermi level.

Fig. 4. Band structure of InSb calculated by the fully relativistic full-potential LCAO method at the experimental lattice constant. The dotted line represents the energy of the top of the valence bands.

Fig. 5. Band structure of InSb calculated by the scalar relativistic full-potential LCAO method at the experimental lattice constant. The dotted line represents the energy of the top of the valence bands.

Fig. 6. Band structure of InSb calculated by the nonrelativistic full-potential LCAO method at the experimental lattice constant. The dotted line represents the energy of the top of the valence bands. 
Table I. Atomic orbitals and number of mesh points used for calculating diatomic molecules with heavy elements.

\begin{tabular}{lcc}
\hline Atom & Atomic orbitals(atomic charge $)^{\text {a })}$ & Number of mesh points \\
\hline $\mathrm{H}$ & $1 s, 2 p($ neutral $) ; 1 s(2+)$ & 2064 \\
$\mathrm{C}$ & $1 s, 2 s, 2 p($ neutral $) ; 2 s, 2 p(2+) ; 3 d(4+)$ & 2064 \\
$\mathrm{O}$ & $1 s, 2 s, 2 p($ neutral $) ; 2 s, 2 p(2+) ; 3 d(6+)$ & 2064 \\
$\mathrm{~F}$ & $1 s, 2 s, 2 p($ neutral $) ; 2 s, 2 p(2+) ; 3 d(7+)$ & 2064 \\
$\mathrm{~S}$ & {$[\mathrm{Ne}] ; 3 s, 3 p($ neutral $) ; 3 s, 3 p, 3 d(2+)$} & 2580 \\
$\mathrm{Cl}$ & {$[\mathrm{Ne}] ; 3 s, 3 p($ neutral $) ; 3 s, 3 p, 3 d(2+)$} & 3096 \\
$\mathrm{Br}$ & {$[\mathrm{Ar}] ; 3 s, 3 p($ neutral $) ; 3 s, 3 p, 3 d(2+)$} & 4128 \\
$\mathrm{Hf}$ & {$[\mathrm{Xe}] ; 4 f, 5 d, 6 s($ neutral $) ; 6 p(1+) ; 5 d, 6 s(2+) ; 6 p(3+)$} & 4128 \\
$\mathrm{Ta}$ & {$[\mathrm{Xe}] ; 4 f, 5 d, 6 s($ neutral $) ; 6 p(1+) ; 5 d, 6 s(2+) ; 6 p(3+)$} & 4128 \\
$\mathrm{Ir}$ & {$[\mathrm{Xe}] ; 4 f, 5 d, 6 s($ neutral $) ; 6 p(1+) ; 5 d, 6 s(2+) ; 6 p(3+)$} & 4128 \\
$\mathrm{Pt}$ & {$[\mathrm{Xe}] ; 4 f, 5 d, 6 s($ neutral $) ; 6 p(1+) ; 5 d, 6 s(2+) ; 6 p(3+)$} & 4128 \\
$\mathrm{Au}$ & {$[\mathrm{Xe}] ; 4 f, 5 d, 6 s($ neutral $) ; 6 p(1+) ; 5 d, 6 s(2+) ; 6 p(3+)$} & 4128 \\
$\mathrm{Hg}$ & {$[\mathrm{Xe}] ; 4 f, 5 d, 6 s($ neutral $) ; 6 p(1+) ; 5 d, 6 s(2+) ; 6 p(3+)$} & 4128 \\
$\mathrm{Tl}$ & {$[\mathrm{Xe}] ; 4 f, 5 d, 6 s, 6 p($ neutral $) ; 6 s, 6 p, 6 d(2+)$} & 4128 \\
$\mathrm{~Pb}$ & {$[\mathrm{Xe}] ; 4 f, 5 d, 6 s, 6 p($ neutral $) ; 6 s, 6 p, 6 d(2+)$} & 4128 \\
$\mathrm{Bi}$ & {$[\mathrm{Xe}] ; 4 f, 5 d, 6 s, 6 p($ neutral $) ; 6 s, 6 p, 6 d(2+)$} & \\
\hline
\end{tabular}

a) $[\mathrm{Ne}],[\mathrm{Ar}]$, and $[\mathrm{Xe}]$ represent $1 s, 2 s, 2 p$ (neutral), $1 s, 2 s, 2 p, 3 s, 3 p$ (neutral), and $1 s, 2 s, 2 p, 3 s, 3 p, 3 d, 4 s, 4 p, 4 d, 5 s, 5 p($ neutral), respectively. 
Table II. Bond lengths in $\AA$ of diatomic molecules with heavy elements. The errors in \% relative to the experimental results are shown in parenthesis.

\begin{tabular}{|c|c|c|c|c|}
\hline Molecule & Expt. $^{a)}$ & FFLCAO $^{\text {b) }}$ & $\mathrm{SFLCAO}^{\mathrm{c}}$ & $\mathrm{NFLCAO}^{\mathrm{d})}$ \\
\hline $\mathrm{HfO}$ & 1.723 & $1.733(+0.6)$ & $1.735(+0.7)$ & $1.765(+2.4)$ \\
\hline $\mathrm{TaO}$ & 1.687 & $1.700(+0.8)$ & $1.701(+0.8)$ & $1.740(+3.1)$ \\
\hline $\mathrm{IrC}$ & 1.683 & $1.673(-0.6)$ & $1.669(-0.8)$ & $1.695(+0.7)$ \\
\hline $\mathrm{PtO}$ & 1.727 & $1.734(+0.4)$ & $1.741(+0.8)$ & $1.907(+10.4)$ \\
\hline $\mathrm{Au}_{2}$ & 2.472 & $2.498(+1.1)$ & $2.498(+1.1)$ & $2.709(+9.7)$ \\
\hline $\mathrm{HgH}$ & 1.745 & $1.744(-0.1)$ & $1.770(+1.4)$ & $1.862(+6.7)$ \\
\hline $\mathrm{TlH}$ & 1.870 & $1.893(+1.2)$ & $1.916(+2.5)$ & $1.945(+4.0)$ \\
\hline $\mathrm{TlF}$ & 2.084 & $2.103(+0.9)$ & $2.105(+1.0)$ & $2.103(+0.9)$ \\
\hline $\mathrm{TlCl}$ & 2.485 & $2.494(+0.4)$ & $2.504(+0.8)$ & $2.516(+1.2)$ \\
\hline $\mathrm{TlBr}$ & 2.618 & $2.637(+0.7)$ & $2.639(+0.8)$ & $2.661(+1.6)$ \\
\hline $\mathrm{PbS}$ & 2.287 & $2.314(+1.2)$ & $2.306(+0.8)$ & $2.332(+2.0)$ \\
\hline $\mathrm{BiO}$ & 1.934 & $1.954(+1.0)$ & $1.932(-0.1)$ & $1.951(+0.9)$ \\
\hline
\end{tabular}
a) Reference 35 .
b) Fully relativistic full-potential LCAO calculations.
c) Scalar relativistic full-potential LCAO calculations.
d) Nonrelativistic full-potential LCAO calculations. 
Table III. Spin polarizations in $\hbar$ of diatomic molecules with heavy elements. The experimental results are deduced from the experimental spin multiplicity in the ground states of the molecules.

\begin{tabular}{lcccc}
\hline Molecule & Expt. $^{\mathrm{a})}$ & FFLCAO $^{\mathrm{b})}$ & SFLCAO $^{\mathrm{c}}$ & NFLCAO $^{\mathrm{d})}$ \\
\hline $\mathrm{HfO}$ & 0 & 0 & 0 & 1 \\
$\mathrm{TaO}$ & $1 / 2$ & 0.49 & $1 / 2$ & $3 / 2$ \\
$\mathrm{IrC}$ & $1 / 2$ & 0.50 & $1 / 2$ & $1 / 2$ \\
$\mathrm{PtO}$ & 0 & 0 & 0 & 1 \\
$\mathrm{Au}$ & 0 & 0 & 0 & 0 \\
$\mathrm{HgH}$ & $1 / 2$ & 0.49 & $1 / 2$ & $1 / 2$ \\
$\mathrm{TlH}$ & 0 & 0 & 0 & 0 \\
$\mathrm{TlF}$ & 0 & 0 & 0 & 0 \\
$\mathrm{TlCl}$ & 0 & 0 & 0 & 0 \\
$\mathrm{TlBr}$ & 0 & 0 & 0 & 0 \\
$\mathrm{PbS}$ & 0 & 0 & 0 & 0 \\
$\mathrm{BiO}$ & $1 / 2$ & 0.46 & $1 / 2$ & $1 / 2$ \\
\hline
\end{tabular}
a) Reference 35 .
b) Fully relativistic full-potential LCAO calculations.
c) Scalar relativistic full-potential LCAO calculations.
d) Nonrelativistic full-potential LCAO calculations. 
Table IV. One-electron energies in eV of the highest-occupied and lowest-unoccupied molecular orbitals of diatomic molecules with heavy elements. The gaps between the two orbitals are also shown.

\begin{tabular}{|c|c|c|c|c|c|c|c|c|c|}
\hline \multirow[t]{2}{*}{ Molecule } & \multicolumn{3}{|c|}{ FFLCAO $^{a)}$} & \multicolumn{3}{|c|}{$\mathrm{SFLCAO}^{\mathrm{b})}$} & \multicolumn{3}{|c|}{$\mathrm{NFLCAO}^{\mathrm{c})}$} \\
\hline & HOMO & LUMO & Gap & HOMO & LUMO & Gap & HOMO & LUMO & Gap \\
\hline $\mathrm{HfO}$ & -5.34 & -3.94 & 1.40 & -5.34 & -3.73 & 1.61 & -3.68 & -3.68 & 0.00 \\
\hline $\mathrm{TaO}$ & -4.94 & -4.43 & 0.51 & -5.07 & -4.30 & 0.77 & -4.47 & -3.24 & 1.23 \\
\hline $\mathrm{IrC}$ & -5.80 & -5.68 & 0.12 & -5.91 & -5.79 & 0.12 & -4.63 & -4.15 & 0.48 \\
\hline $\mathrm{PtO}$ & -6.55 & -6.22 & 0.33 & -6.43 & -6.43 & 0.00 & -5.22 & -5.22 & 0.00 \\
\hline $\mathrm{Au}_{2}$ & -6.14 & -4.74 & 1.40 & -6.51 & -4.67 & 1.84 & -5.20 & -3.30 & 1.90 \\
\hline $\mathrm{HgH}$ & -4.89 & -3.92 & 0.97 & -4.87 & -3.85 & 1.02 & -4.43 & -3.59 & 0.84 \\
\hline $\mathrm{TlH}$ & -5.06 & -2.90 & 2.16 & -4.89 & -2.56 & 2.33 & -4.82 & -2.80 & 2.02 \\
\hline $\mathrm{TlF}$ & -7.00 & -2.96 & 4.04 & -6.91 & -2.45 & 4.46 & -6.50 & -2.70 & 3.80 \\
\hline $\mathrm{TlCl}$ & -6.78 & -3.17 & 3.61 & -6.70 & -2.66 & 4.04 & -6.36 & -2.87 & 3.49 \\
\hline $\mathrm{TlBr}$ & -6.40 & -3.19 & 3.21 & -6.46 & -2.70 & 3.76 & -6.25 & -2.91 & 3.34 \\
\hline $\mathrm{PbS}$ & -6.16 & -3.77 & 2.39 & -6.24 & -3.34 & 2.90 & -6.41 & -3.55 & 2.86 \\
\hline $\mathrm{BiO}$ & -4.97 & -4.54 & 0.43 & -4.57 & -4.57 & 0.00 & -4.76 & -4.76 & 0.00 \\
\hline
\end{tabular}

a) Fully relativistic full-potential LCAO calculations.

b) Scalar relativistic full-potential LCAO calculations.

c) Nonrelativistic full-potential LCAO calculations. 
Table V. Dissociation energies in eV of diatomic molecules with heavy elements. The errors in $\%$ relative to the experimental results are shown in parenthesis.

\begin{tabular}{|c|c|c|c|c|}
\hline Molecule & Expt. $^{\text {a) }}$ & FFLCAO $^{\mathrm{b})}$ & $\mathrm{SFLCAO}^{\mathrm{c})}$ & $\mathrm{NFLCAO}^{\mathrm{d})}$ \\
\hline $\mathrm{HfO}$ & 8.19 & $10.10(+23)$ & $10.57(+30)$ & $10.05(+23)$ \\
\hline $\mathrm{TaO}$ & 8.2 & $9.86(+20)$ & $10.40(+27)$ & $10.60(+29)$ \\
\hline $\mathrm{IrC}$ & 6.45 & $8.25(+28)$ & $9.02(+40)$ & $10.95(+70)$ \\
\hline $\mathrm{PtO}$ & 3.82 & $5.60(+47)$ & $5.43(+42)$ & $5.13(+34)$ \\
\hline $\mathrm{Au}_{2}$ & 2.30 & $2.89(+26)$ & $2.83(+23)$ & $1.99(-13)$ \\
\hline $\mathrm{HgH}$ & 0.37 & $0.82(+122)$ & $0.75(+103)$ & $1.14(+208)$ \\
\hline $\mathrm{TlH}$ & 1.97 & $2.44(+24)$ & $2.96(+50)$ & $2.97(+51)$ \\
\hline $\mathrm{TlF}$ & 4.57 & $5.48(+20)$ & $6.16(+35)$ & $6.30(+38)$ \\
\hline $\mathrm{TlCl}$ & 3.82 & $4.33(+13)$ & $4.99(+31)$ & $5.00(+31)$ \\
\hline $\mathrm{TlBr}$ & 3.42 & $3.86(+13)$ & $4.58(+34)$ & $4.58(+34)$ \\
\hline $\mathrm{PbS}$ & 3.49 & $4.41(+26)$ & $5.74(+55)$ & $5.79(+66)$ \\
\hline $\mathrm{BiO}$ & 3.47 & $4.92(+42)$ & $5.40(+56)$ & $5.59(+61)$ \\
\hline
\end{tabular}
a) Reference 35 .
b) Fully relativistic full-potential LCAO calculations.
c) Scalar relativistic full-potential LCAO calculations.
d) Nonrelativistic full-potential LCAO calculations. 
Table VI. Lattice constant in $\AA$ and bulk modulus in GPa of Au. The errors in $\%$ relative to the experimental results are shown in parenthesis.

Lattice constant

\begin{tabular}{lcc}
\hline Expt. $^{\mathrm{a})}$ & 4.078 & 173.2 \\
FFLCAO $^{\mathrm{b})}$ & $4.061(-0.4)$ & $182(+5)$ \\
SFLCAO $^{\mathrm{c}}$ & $4.076(0.0)$ & $189(+9)$ \\
NFLCAO $^{\mathrm{d})}$ & $4.286(+5.1)$ & $114(-34)$ \\
\hline Ref. $39^{\mathrm{e})}$ & $4.050(-0.7)$ & $190(+10)$ \\
Ref. $40^{\mathrm{f})}$ & $4.057(-0.5)$ & $198(+14)$ \\
Ref. $41^{\mathrm{g})}$ & $4.053(-0.6)$ & $205(+18)$ \\
Ref. $42^{\mathrm{h})}$ & $4.059(-0.5)$ & $168.9(-2)$ \\
Ref. $43^{\mathrm{i})}$ & $4.069(-0.2)$ & $182(+5)$ \\
Ref. $44^{\mathrm{j})}$ & $4.070(-0.2)$ & $193(+11)$ \\
Ref. $4^{\mathrm{k})}$ & $4.064(-0.3)$ & $196(+13)$ \\
Ref. $17^{\mathrm{l})}$ & $4.039(-1.0)$ & $112(-35)$ \\
Ref. $17^{\mathrm{m})}$ & $4.274(+4.8)$ & $(-2)$ \\
\hline
\end{tabular}

a) Reference 38 .

b) Fully relativistic full-potential LCAO calculations.

c) Scalar relativistic full-potential LCAO calculations.

d) Nonrelativistic full-potential LCAO calculations.

e) Fully relativistic full-potential KKR calculations.

f) Scalar relativistic augmented-spherical-wave calculations.

g) Scalar relativistic full-potential linearized APW calculations.

h) Scalar relativistic full-potential linearized APW calculations.

i) Scalar relativistic APW calculations.

j) Pseudopotential mixed-basis calculations.

k) Scalar relativistic full-potential LMTO calculations.

l) Scalar relativistic full-potential LCAO calculations.

m) Nonrelativistic full-potential LCAO calculations.
Bulk modulus

$$
\begin{aligned}
& 182(+5) \\
& 189(+9) \\
& 114(-34) \\
& 190(+10) \\
& 198(+14) \\
& 205(+18) \\
& 168.9(-2) \\
& 182(+5) \\
& 193(+11) \\
& 196(+13)
\end{aligned}
$$


Table VII. $d$-band width in $\mathrm{eV}$ of $\mathrm{Au}$.

$d$-band width

FFLCAO $^{a)}$

6.28

SFLCAO $^{\text {b) }}$

5.55

$\mathrm{NFLCAO}^{\mathrm{c})}$

5.02

Ref. $39^{\mathrm{d})}$

6.15

Ref. $45^{\mathrm{e})}$

6.31

Ref. $43^{\text {f) }}$

5.89

Ref. $17^{\mathrm{g})}$

5.85

Ref. $17^{\mathrm{h}}$

5.01

a) Fully relativistic full-potential LCAO calculations.

b) Scalar relativistic full-potential LCAO calculations.

c) Nonrelativistic full-potential LCAO calculations.

d) Fully relativistic full-potential KKR calculations.

e) Scalar relativistic APW calculations with the spin-orbit coupling.

f) Scalar relativistic APW calculations without the spin-orbit coupling.

g) Scalar relativistic full-potential LCAO calculations without the spin-orbit coupling.

h) Nonrelativistic full-potential LCAO calculations. 
Table VIII. Cohesive energy in eV/atom of Au. The errors in \% relative to the experimental result are shown in parenthesis.

\begin{tabular}{|c|c|c|c|c|}
\hline & Expt. ${ }^{a)}$ & FFLCAO $^{\mathrm{b})}$ & SFLCAO $^{\mathrm{c})}$ & $\mathrm{NFLCAO}^{\mathrm{d})}$ \\
\hline $\mathrm{Au}$ & 3.79 & $4.39(+16)$ & $4.19(+11)$ & $3.39(+11)$ \\
\hline
\end{tabular}

a) Calculated from standard heat of formation at $298.15 \mathrm{~K}$.

b) Fully relativistic full-potential LCAO calculations.

c) Scalar relativistic full-potential LCAO calculations.

d) Nonrelativistic full-potential LCAO calculations. 
Table IX. Lattice constant in $\AA$ and bulk modulus in GPa of InSb. The errors in \% relative to the experimental results are shown in parenthesis.

\begin{tabular}{lcc}
\hline & Lattice constant & Bulk modulus \\
\hline Expt. $^{\text {a) }}$ & 6.479 & 48.3 \\
FFLCAO $^{\text {b) }}$ & $6.463(-0.2)$ & $48(0)$ \\
SFLCAO $^{\text {c }}$ & $6.488(+0.1)$ & $48(0)$ \\
NFLCAO $^{\text {d) }}$ & $6.542(+1.0)$ & $53(+10)$ \\
\hline Ref. $39^{\mathrm{e})}$ & $6.448(-0.5)$ & $45(-7)$ \\
Ref. $3^{\text {f) }}$ & $6.464(-0.2)$ & $48(0)$ \\
Ref. $3^{\mathrm{g})}$ & $6.36(-1.8)$ & $48(0)$ \\
Ref. $46^{\mathrm{h})}$ & $6.359(-1.9)$ & $47(-3)$ \\
\hline
\end{tabular}
a) Reference 38 .
b) Fully relativistic full-potential LCAO calculations.
c) Scalar relativistic full-potential LCAO calculations.
d) Nonrelativistic full-potential LCAO calculations.
e) Fully relativistic full-potential KKR calculations.
f) Scalar relativistic full-potential linearized APW calculations.
g) Pseudopotential plane-wave calculations.
h) Pseudopotential plane-wave calculations. 
Table X. One-electron energies in $\mathrm{eV}$ of $\operatorname{InSb}$ at $\Gamma, X$, and $L$.

\begin{tabular}{|c|c|c|c|c|c|c|}
\hline & FFLCAO $^{a)}$ & SFLCAO $^{\mathrm{b})}$ & $\mathrm{NFLCAO}^{\mathrm{c})}$ & Ref. $39^{\mathrm{d})}$ & Ref. $3^{\mathrm{e})}$ & Ref. $3^{\mathrm{f})}$ \\
\hline \multirow[t]{2}{*}{$\Gamma_{15 c}$} & 2.82 & 2.95 & 2.89 & 2.83 & & \\
\hline & 2.38 & & & 2.39 & & \\
\hline$\Gamma_{1 c}$ & -0.65 & -0.38 & 1.00 & -0.66 & -0.74 & -0.47 \\
\hline \multirow[t]{2}{*}{$\Gamma_{15 v}$} & 0.00 & 0.00 & 0.00 & 0.00 & 0.00 & 0.00 \\
\hline & -0.78 & & & -0.77 & -0.76 & \\
\hline$\Gamma_{1 v}$ & -11.06 & -10.81 & -10.08 & -11.05 & -11.11 & -10.83 \\
\hline$\Gamma_{12 v}$ & -14.42 & -14.44 & -15.49 & -14.26 & & -14.64 \\
\hline \multirow[t]{2}{*}{$\Gamma_{25 v}$} & -14.48 & -14.54 & -15.57 & -14.34 & & -14.73 \\
\hline & -15.29 & & & -15.15 & & \\
\hline$X_{3 c}$ & 0.99 & 1.25 & 1.41 & 0.94 & & \\
\hline$X_{1 c}$ & 0.98 & 1.24 & 1.31 & 0.91 & 1.04 & 1.28 \\
\hline \multirow[t]{2}{*}{$X_{5 v}$} & -2.46 & -2.28 & -2.28 & -2.49 & -2.48 & -2.28 \\
\hline & -2.64 & & & -2.67 & -2.65 & \\
\hline$X_{3 v}$ & -6.29 & -6.01 & -5.58 & -6.24 & -6.29 & -6.01 \\
\hline$X_{1 v}$ & -9.08 & -8.85 & -7.96 & -9.05 & -9.15 & -8.88 \\
\hline \multirow[t]{2}{*}{$L_{3 c}$} & 3.62 & 3.80 & 3.69 & 3.51 & & \\
\hline & 3.42 & & & 3.33 & & \\
\hline$L_{1 c}$ & 0.15 & 0.42 & 1.08 & 0.15 & 0.13 & 0.39 \\
\hline \multirow[t]{2}{*}{$L_{3 v}$} & -1.04 & -1.02 & -1.01 & -1.05 & -1.06 & -1.04 \\
\hline & -1.53 & & & -1.53 & -1.54 & \\
\hline$L_{1 v}$ & -5.98 & -5.71 & -5.39 & -5.93 & -5.98 & -5.70 \\
\hline$L_{1 v}$ & -9.69 & -9.46 & -8.63 & -9.68 & -9.75 & -9.48 \\
\hline
\end{tabular}

a) Fully relativistic full-potential LCAO calculations.

b) Scalar relativistic full-potential LCAO calculations.

c) Nonrelativistic full-potential LCAO calculations.

d) Fully relativistic full-potential KKR calculations.

e) Scalar relativistic full-potential linearized APW calculations with the spin-orbit coupling.

f) Scalar relativistic full-potential linearized APW calculations without the spin-orbit coupling. 
Table XI. Cohesive energy in eV/atom of InSb. The errors in \% relative to the experimental result are shown in parenthesis.

\begin{tabular}{|c|c|c|c|c|}
\hline & Expt. ${ }^{a)}$ & FFLCAO $^{\text {b) }}$ & SFLCAO $^{\mathrm{c})}$ & $\mathrm{NFLCAO}^{\mathrm{d})}$ \\
\hline $\mathrm{InSb}$ & 2.80 & $2.79(0)$ & $3.21(+15)$ & $4.27(+53)$ \\
\hline
\end{tabular}

a) Calculated from standard heat of formation at $298.15 \mathrm{~K}$.

b) Fully relativistic full-potential LCAO calculations.

c) Scalar relativistic full-potential LCAO calculations.

d) Nonrelativistic full-potential LCAO calculations. 


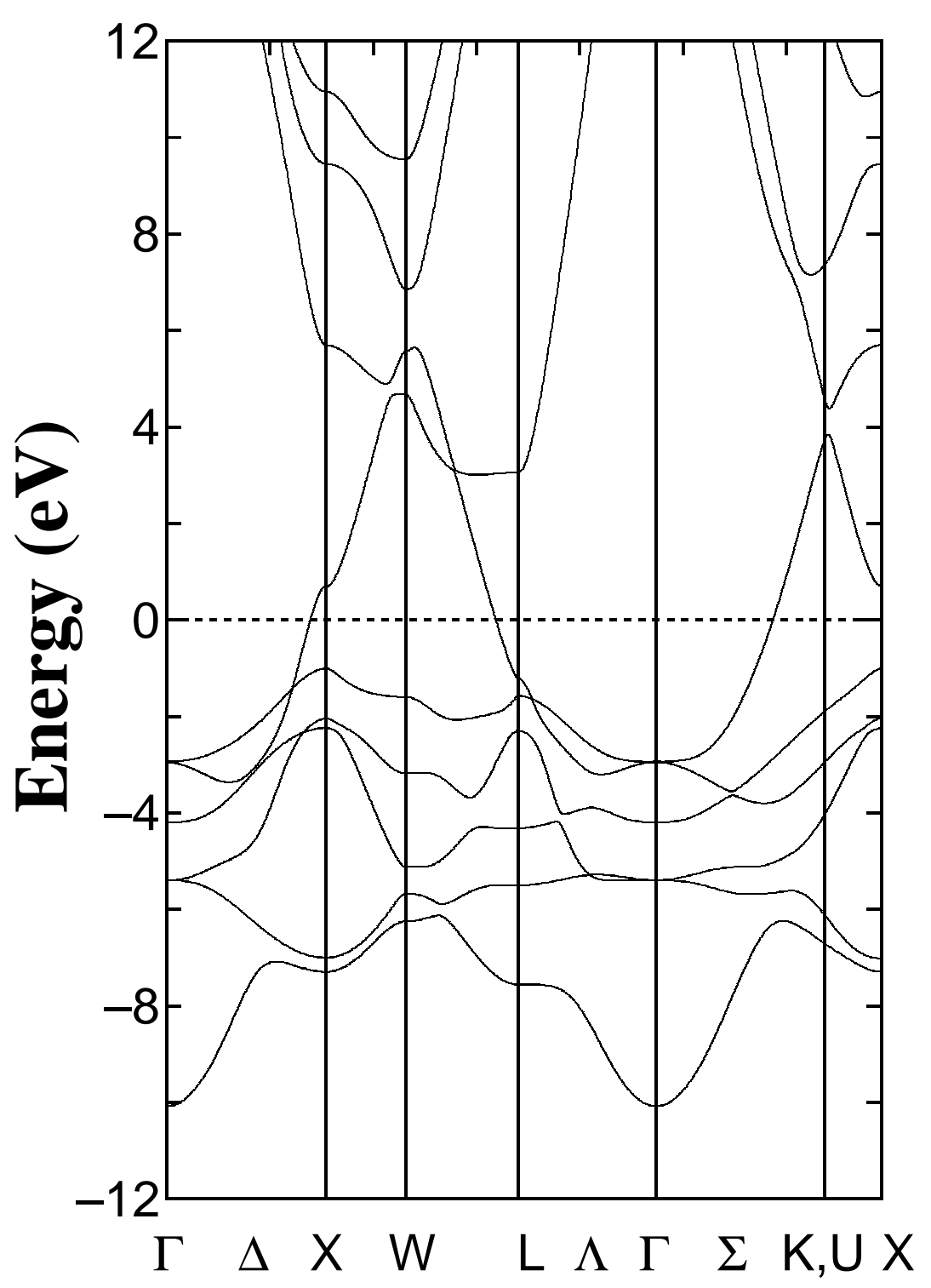

Fig. 1 


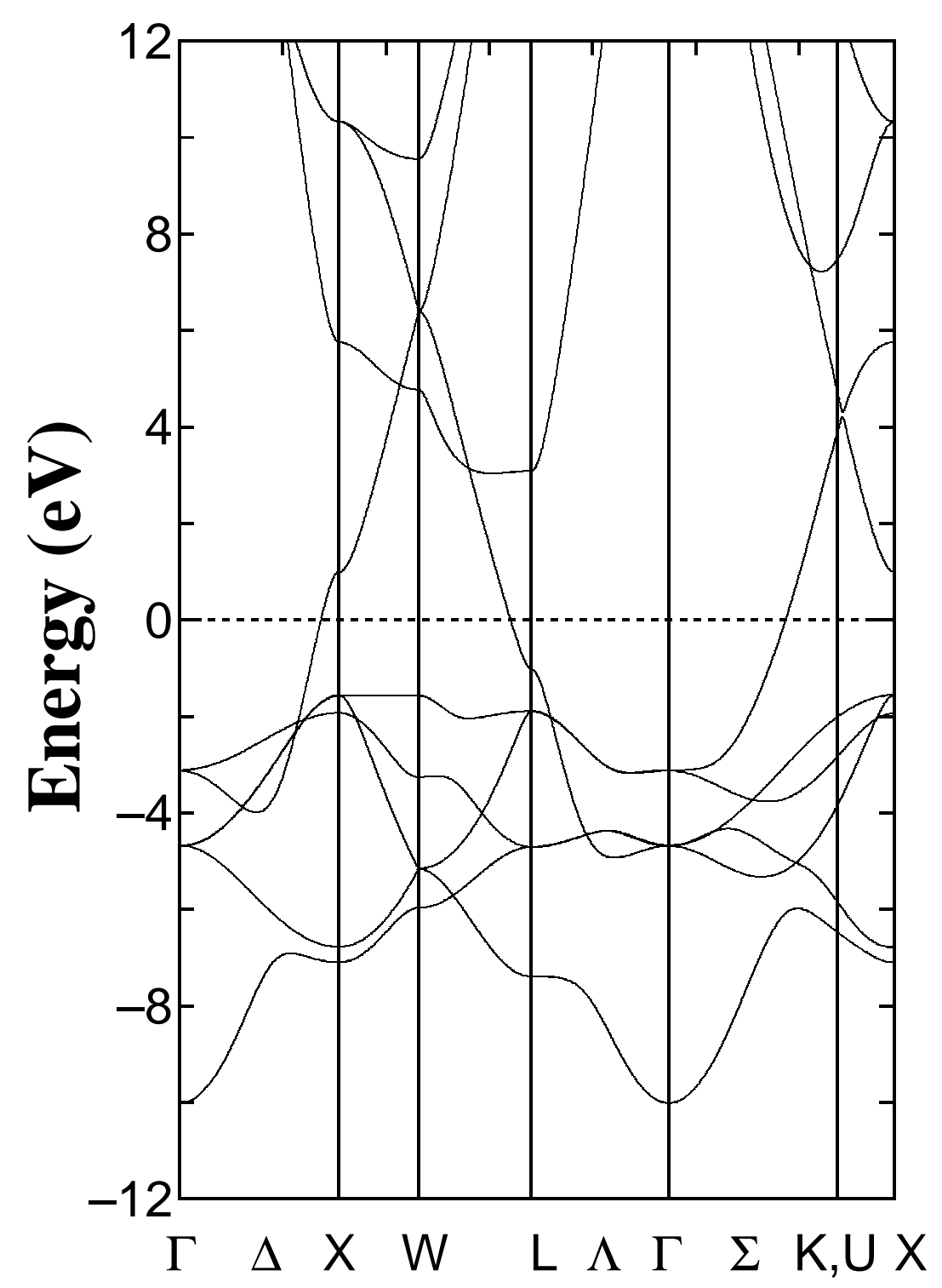

Fig. 2 


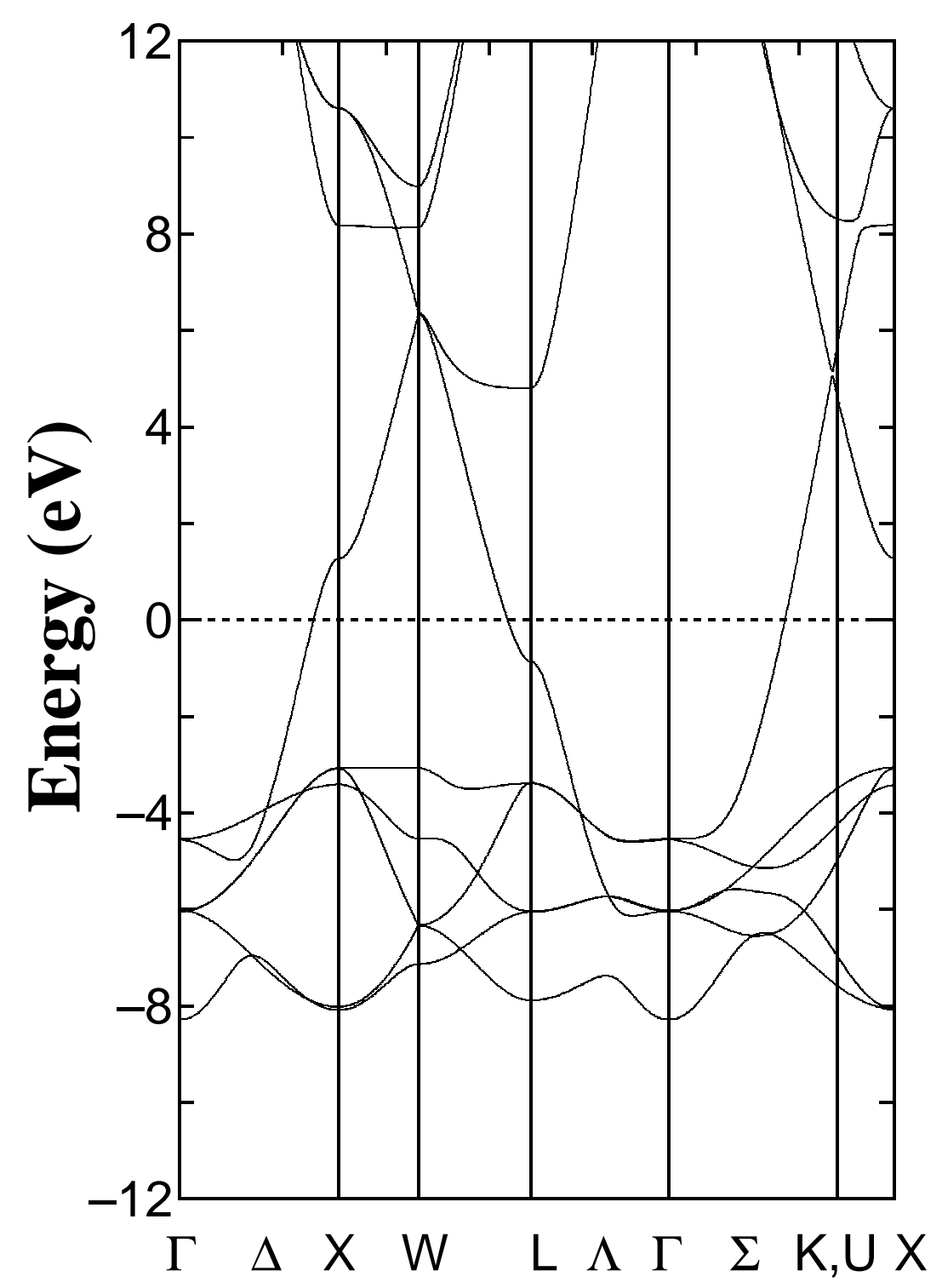

Fig. 3 


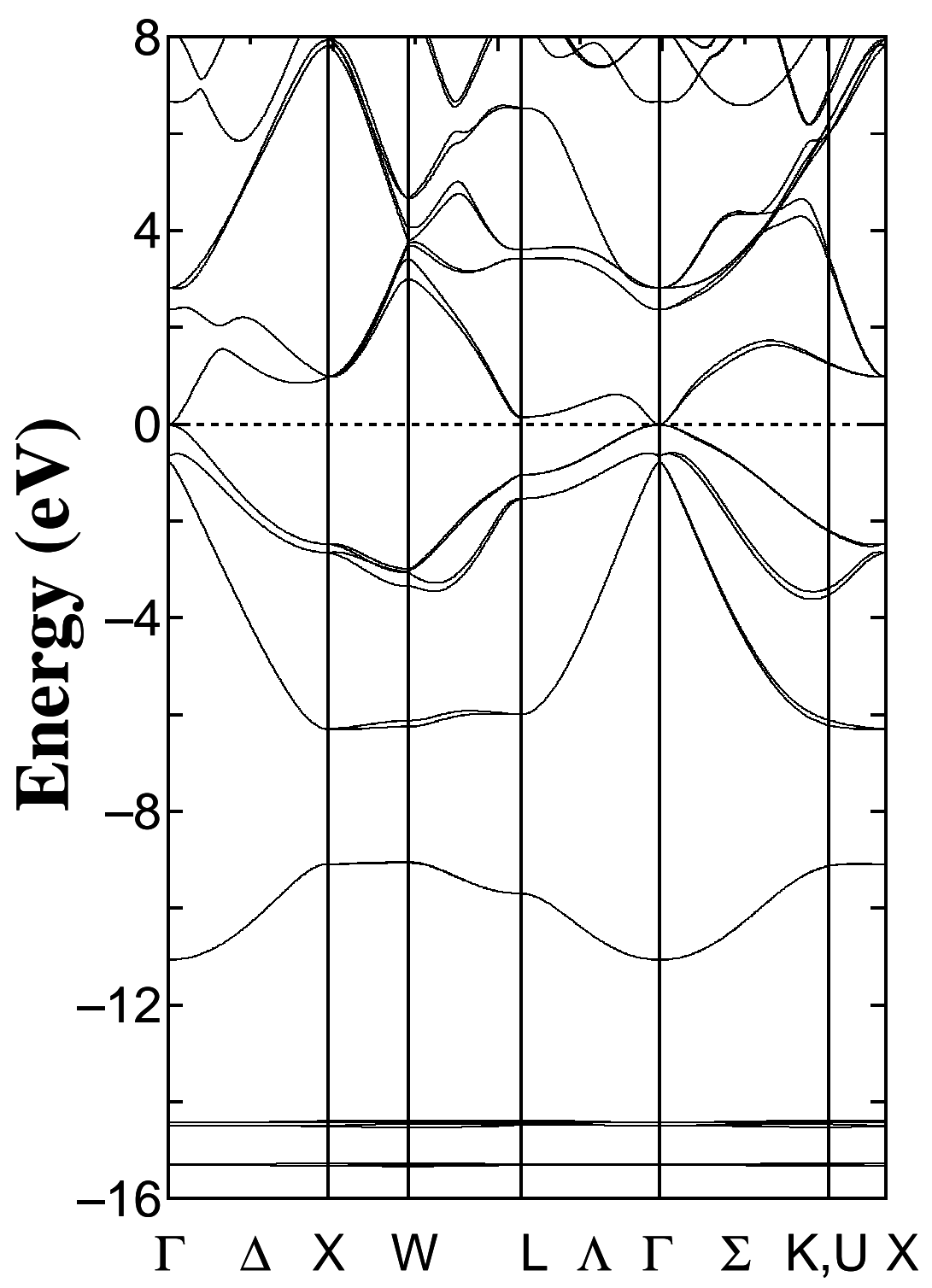

Fig. 4 


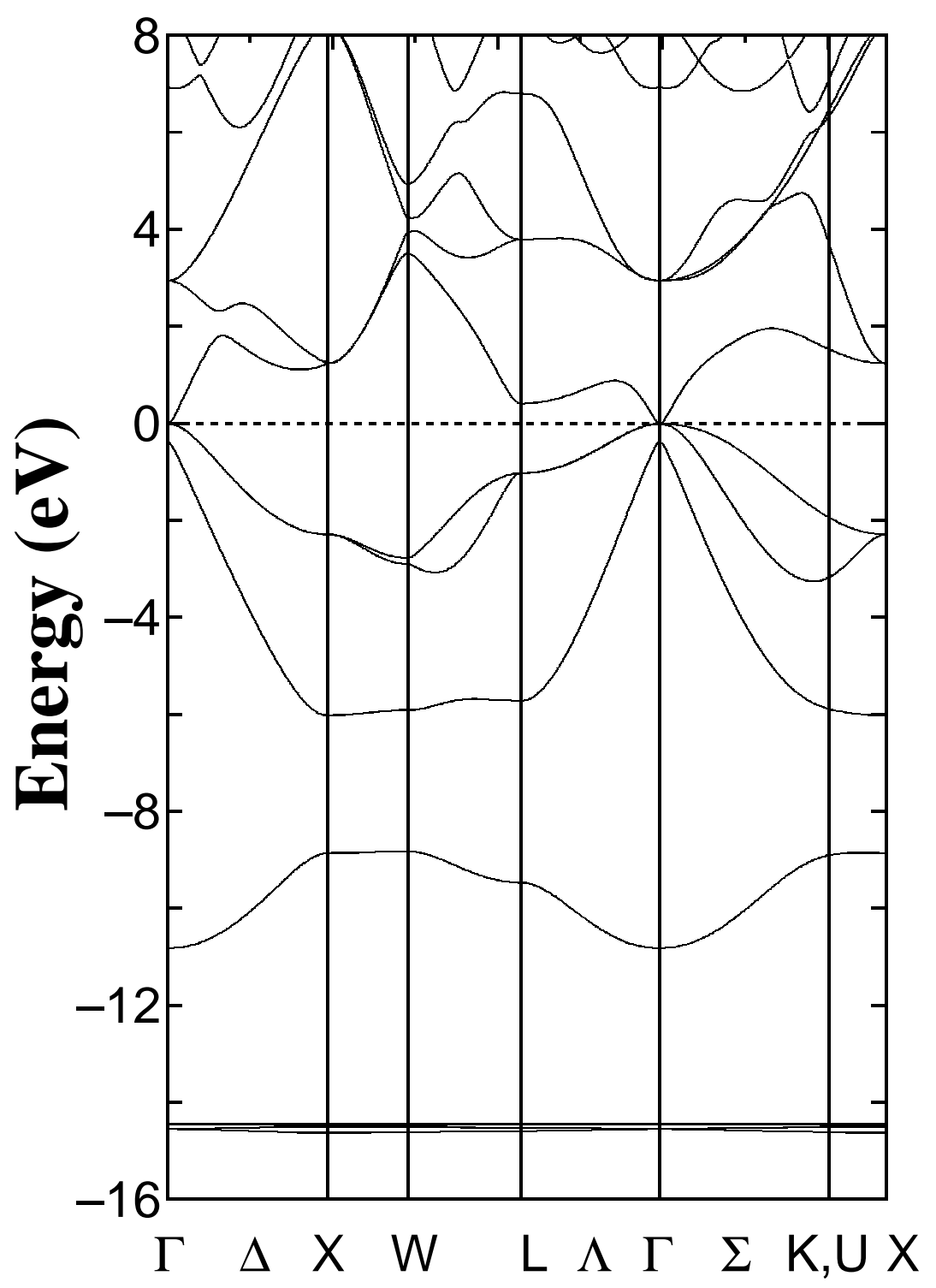

Fig. 5 


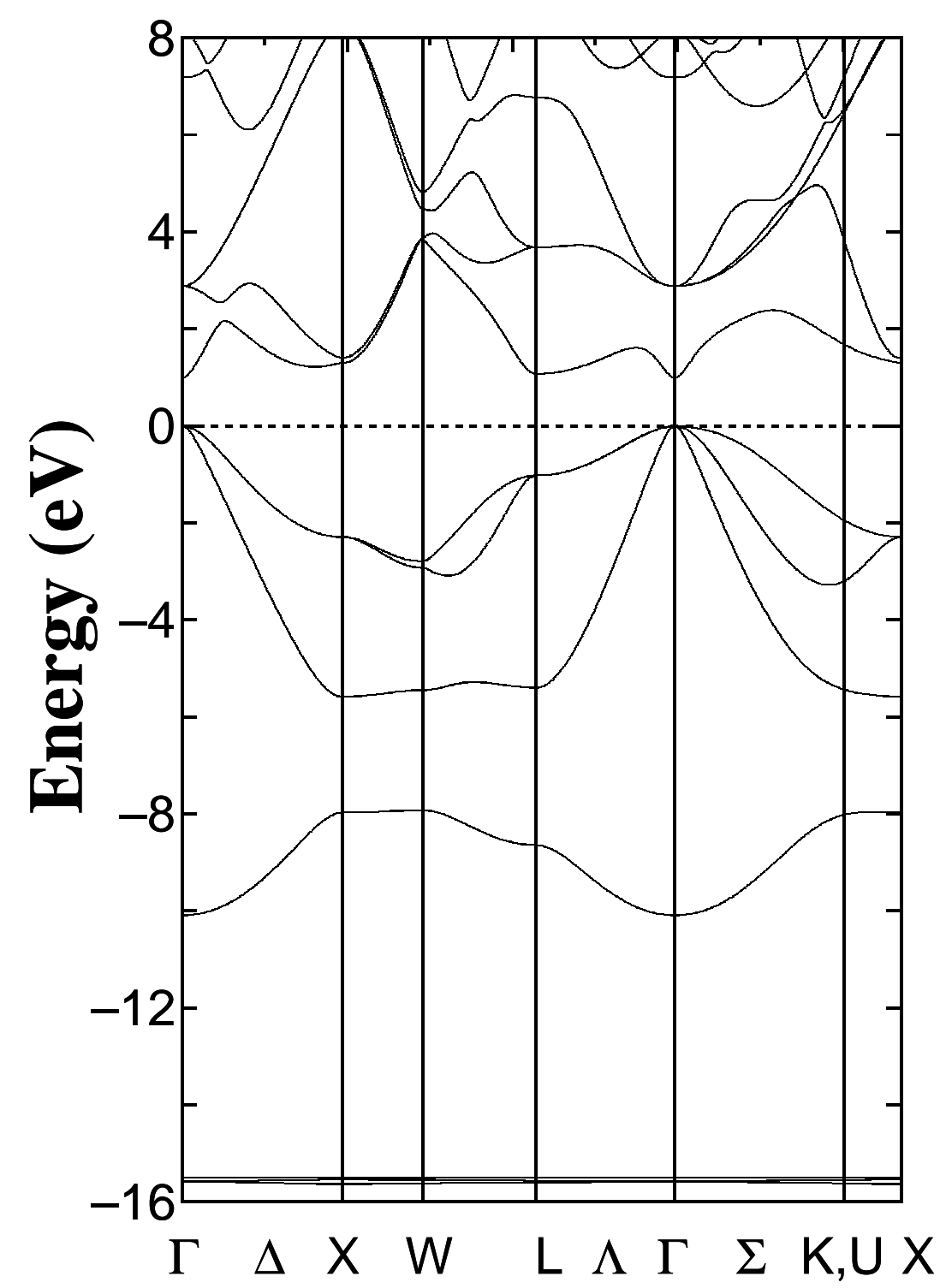

Fig. 6 